\title{
Photophysical and Thermodynamic Parameters of Hematoporphyrin in Solutions and Monolayers
}

\author{
M. Kotkowiak · J. Lukasiewicz • A. Dudkowiak
}

Received: 14 April 2012 / Accepted: 26 September 2012 / Published online: 10 October 2012 (C) The Author(s) 2012. This article is published with open access at Springerlink.com

\begin{abstract}
To achieve better understanding of anticancer photosensitizing efficiency and cellular membrane location ability, a study of hematoporphyrin and phospholipids in a dye and in a mixture of a dye and lipids in volumetric and 2D systems was performed. By means of steady-state absorption, fluorescence, and time-resolved optoacoustic spectroscopy, the fluorescence quantum yield, the fraction of absorbed energy converted into heat on a subnanosecond timescale, the efficiency of the dye's triplet-state population, and singlet oxygen generation characteristics of the dye were determined in chosen organic solvents. On the basis of the isotherms of hematoporphyrin and its mixture with phospholipid in Langmuir monolayers, the excess area, excess Gibbs energy, and compression modulus were estimated and the molecular interactions in the model systems investigated were discussed.
\end{abstract}

Keywords Hematoporphyrin - Monolayer · Optical and photothermal spectroscopy

\section{Introduction}

The molecular structure of porphyrins to a high degree determines their ability to penetrate and interact with amphiphilic and anisotropic cell membranes [1,2]. Investigation of certain biological models helps the selection of better sensitizers for biomedical purposes including photodynamic therapy (PDT) of cancer and its diagnosis.

Hematoporphyrin (HP), an iron-free derivative of heme, was the first commercial but not perfect photosensitizing substance used in clinical treatment $[3,4]$. The effectiveness of $\mathrm{HP}$ and its derivatives acting as photosensitizers is known to depend on the

\footnotetext{
M. Kotkowiak · J. Łukasiewicz · A. Dudkowiak $(\varangle)$

Faculty of Technical Physics, Poznan University of Technology, Nieszawska 13a,

60-965 Poznan, Poland

e-mail: alina.dudkowiak@put.poznan.pl
} 
aggregation form of the dye $[5,6]$ and its localization in pathologically changed cells $[7,8]$.

Important properties of sensitizer molecules, such as the wavelength of absorption, yields of triplet-state formation, and singlet oxygen generation determine the dye phototoxicity and considerably change according to micro-environment interactions [9]. Recently, it has been shown that an understanding of the interactions between the photosensitizer and components of the biological membranes such as lipids is essential for PDT effectiveness [10,11].

The results of our experiments permitted estimation of photophysical and thermodynamic parameters of HP and its mixture with phospholipids in organic solvents whose presence can modify the dye-solvent molecular interactions with the environment, and so also in a 2D monolayer at the air-water interface, which can be treated as a model of a biological membrane. The Langmuir method of the production of monolayers on the water subphase permits investigation of the incorporation and interaction of the photoactive molecules in biological membranes [10], determining their in vivo activity.

This study was undertaken to check the influence of the environment on the photophysical and thermodynamic parameters of HP in volumetric and 2D model systems to better understand its photosensitizing mechanism. By means of steady-state absorption, fluorescence, and time-resolved laser-induced optoacoustic spectroscopy (LIOAS), characterization of HP was performed. Photophysical properties of a dye, such as the aggregation state, efficiency of fluorescence, intersystem crossing, and energy transfer to oxygen were established. In addition, the aim of this study was to identify the interactions between HP and phospholipids to develop the strategies in PDT based on the incorporation of photoactive dyes into unilamellar membranes of water-soluble dye carriers. Therefore, the thermodynamic properties of HP in a dye and mixed dye-phospholipid monolayers were studied. The analysis performed of the surface pressure versus mean molecular area curves, i.e., $(\pi-A)$ isotherms provided information about miscibility, stability, phase transitions, and organization of the molecules investigated.

\section{Measurements}

Hematoporphyrin, a dye of purity $\geq 60 \%$ HPLC was purchased from Fluka, and synthetic phospholipids (DPPC), $99 \%$ pure, were purchased from Sigma-Aldrich. These components were used without further purification.

For estimation of the fluorescence yield for HP dissolved in chosen organic solvents, absorption and fluorescence spectra were measured on a Cary 4000 and a Hitachi F4500 spectrophotometers, respectively. As a fluorescence reference, chlorophyll $a$ was used. By means of the LIOAS method, time-resolved photothermal signals were recorded. Technical specifications and more details of LIOAS apparatus were described elsewhere [12]. For LIOAS experiments, HP was dissolved in acetone, ethanol, and methanol (purchased from $\mathrm{POCH}$, Poland). A calorimetric reference was ferrocene (FC), purchased from Sigma-Aldrich, chosen because of its spectral properties. All measurements were carried out at ambient temperature; additionally, LIOAS measurements were performed in an air and argon atmosphere. 
One- or two-component monolayers were prepared by spreading dye or lipids and their mixtures (v:v) for different molar fractions (MF) from chloroform solutions on the pure water subphase $(\mathrm{pH} 5.3$ and resistivity $18.2 \mathrm{M} \Omega \cdot \mathrm{cm})$. The stock concentrations of the compounds used to prepare the mixtures was $0.1 \mathrm{mM}$. Compression $(\pi-A)$ isotherms were recorded by means of a KSV-2000 system. All monolayer experiments were carried out in dim light. The surface pressure was measured indirectly using a Wilhelmy plate. Monolayers were compressed symmetrically using hydrophilic barriers at a constant rate of $5 \mathrm{~mm} \cdot \mathrm{min}^{-1}$. Each isotherm presented is an average curve collected from three independent measurements.

On the basis of the isotherms, the thermodynamic parameters [13] were calculated, such as: the excess area, the excess Gibbs energy (to analyze the miscibility and interactions between components of the formed monolayer), and the compression modulus (in order to understand phase transitions and isolation and description of molecular interactions). These parameters are defined below.

The excess area ( $\triangle A$ ) was determined using Eq. 1 and the additivity rule (Eq. 2):

$$
\begin{aligned}
& \Delta A=A_{12}-A_{12}^{\mathrm{T}}, \\
& A_{12}^{\mathrm{T}}=X_{1} A_{1}+X_{2} A_{2} .
\end{aligned}
$$

$A_{12}$ represents the experimental values of mean area per molecule in the bicomponent monolayer, $A_{12}^{\mathrm{T}}$ represents the mean area per molecule in an ideal mixture, $A_{i}$ represents the mean area per molecule of the $i$ th component monolayer, and $X_{i}$ is the mole fraction of the $i$ th component.

The compression modulus $\left(c^{-1}\right)$ was determined from $(\pi-A)$ isotherms and was calculated from the relationship:

$$
c^{-1}=-A\left(\frac{\partial \pi}{\partial A}\right) .
$$

The excess Gibbs energy ( $\Delta G)$, of binary mixed monolayers, which represents generation of energy related to the deviation from ideality of the mixture, was calculated as

$$
\begin{aligned}
\Delta G & =\int_{\pi \rightarrow \pi_{0}}^{\pi} A_{12} \mathrm{~d} \pi-\int_{\pi \rightarrow \pi_{0}}^{\pi} A_{12}^{\mathrm{T}} \mathrm{d} \pi \\
& =\int_{\pi \rightarrow \pi_{0}}^{\pi} A_{12} \mathrm{~d} \pi-\left(X_{1} \int_{\pi \rightarrow \pi_{0}}^{\pi} A_{1} \mathrm{~d} \pi+X_{2} \int_{\pi \rightarrow \pi_{0}}^{\pi} A_{2} \mathrm{~d} \pi\right) .
\end{aligned}
$$

\section{Results}

From the absorption and fluorescence spectra (not shown), it follows that both the shape and position of the band assigned to HP are not affected by the formation of porphyrin aggregates, but only slightly affected by the solvent's nature (i.e., peak 
positions varied by approximately $2 \mathrm{~nm}$ to $3 \mathrm{~nm}$ ). The aggregation process of HP, observed as a blue-shift of the Soret band position, occurs extensively for the dye in aqueous solutions; therefore, in this study for spectroscopic measurements, only organic solvents were chosen. The polarity of the solvents (the dielectric constant in the range of 20.7 to 32.6) and the low absorbance used at the excitation wavelength (dye concentration approximately $10^{-6} \mathrm{M}$ ) should prevent aggregate formation. It is known [14] that the intersystem crossing transition occurs less efficiently in the HP aggregates than in monomers and causes a decrease in the efficiency of $T_{1}$ formation ( $\Phi_{\mathrm{T}}$ values).

To obtain the information about the dye photoactivity, the fluorescence yield was calculated and the LIOAS method was used. Analysis of the LIOAS signal gives information on the non-radiative fast deactivation of the excited singlet states, on population of the dye long-lived states, and on the dye ability to generate the reactive oxygen species [12]. The photophysical parameters obtained are given in Table 1. According to these data, an appreciable fraction $(\alpha)$ of the whole energy adsorbed is exchanged into heat with involvement of the singlet state ( $71 \%$ to $80 \%$ ) in the nanosecond time range (below $200 \mathrm{~ns}$ ). This process competes with light emission and the $S_{1} \rightarrow T_{1}$ transition. Due to low efficiency of the former, the effectiveness of triplet generation was about $53 \%$ to $55 \%$. These values were obtained taking into account the known energy of the triplet state $\left(E_{\mathrm{T}}=155.4 \mathrm{~kJ} \cdot \mathrm{mol}^{-1}\right)$ [15]. In deoxygenated solutions, the porphyrin triplet state generally has a lifetime of several hundred microseconds. Molecular oxygen strongly reduces the lifetime of the triplet stage with the biomolecular quenching constant typical of a diffusion-controlled process (i.e., (1 to 2) $\times 10^{9} \mathrm{M}^{-1} \cdot \mathrm{s}^{-1}$ ) [14]. Hence, the observed fraction $\left(S_{\Delta}\right)$ of the triplet state quenched by oxygen is higher than 0.98 in air equilibrated solutions (Table 1). The results for protic solvents are slightly overestimated as compared to those in aprotic solvents.

Literature values for the quantum yield of singlet oxygen generation by HP vary over wide ranges, from 0.13 to 0.42 in aqueous solutions and from 0.43 to 0.93 in organic solvents $[5,16,17]$. These large differences may be due to the experimental conditions, which play a substantial role in estimation of porphyrin cytotoxicity mediated by singlet oxygen, e.g., water/buffered solutions or high concentrated sample, so they can induce dye aggregation. For example, $\Phi_{\Delta}$ in methanol reported for HP changes from 0.93 to $0.65[3,5,6,14,16,17]$ and the latter value is in a good agreement with that obtained by photothermal measurements (Table 1). Analysis of the results confirms high efficiency of triplet-triplet energy transfer between HP and oxygen, independent of the micro-environment, so it can be expected also for HP in lipid moiety.

Langmuir monolayers may be considered as model systems of liposome-type drug carriers or even cell membranes [10]. It is known that HP easily forms aggregates; hence, in order to overcome this effect and supply monomeric HP in the formulation for clinical use, it is necessary to look for appropriate carriers. Liposomes can substantially reduce dye aggregation, preserving its pharmacological action [13] and finally they can selectively deliver the active photosensitizers to hyperproliferating tissues. By using the monolayer technique, it is possible to reproduce and investigate the arrangement at the water subface of the lipid molecules that form the liposomal coating with incorporating and interacting HP molecules. 
Table 1 Some photophysical parameters estimated for HP in organic solvents

\begin{tabular}{lllllll}
\hline Solvent & Atmosphere & $\alpha( \pm 0.02)$ & $\Phi_{\mathrm{F}}$ & $\Phi_{\mathrm{T}}$ & $\Phi_{\Delta}$ & $S_{\Delta}$ \\
\hline Acetone & Air & 0.77 & 0.04 & 0.55 & 0.64 & 1.16 \\
& Argon & 0.71 & & & & \\
\multirow{2}{*}{ Ethanol } & Air & 0.78 & 0.07 & 0.53 & 0.54 & 1.02 \\
& Argon & 0.72 & & & 0.52 & 0.98 \\
Methanol & Air & 0.80 & 0.05 & 0.53 & 0.52 \\
& Argon & 0.72 & & & & \\
\hline
\end{tabular}

$\alpha$, fraction of excitation energy exchange into heat promptly (in shorter time than time resolution of apparatus); $\Phi_{\mathrm{F}}$, fluorescence yield, $\Phi_{\mathrm{F}}=\Phi_{\mathrm{R}} \frac{I}{I_{\mathrm{R}}} \frac{\mathrm{OD}}{\mathrm{OD}} \frac{n^{2}}{n_{\mathrm{R}}^{2}}$, where $\Phi_{\mathrm{R}}$ is the fluorescence quantum yield of the reference. $I, I_{\mathrm{R}} ; \mathrm{OD}, \mathrm{OD}_{\mathrm{R}}$; and $n, n_{\mathrm{R}}$ are the areas under the fluorescence curves, the absorption intensities and refractive indices of the sample and reference, respectively; $\Phi_{\mathrm{T}}$, is the yield of dye's triplet generation calculated using $\Phi_{\mathrm{T}}=\frac{E_{h v}}{E_{\mathrm{T}}}\left(1-\alpha^{A r}\right)$ where $E_{h v}$ is the molar energy of the incident photons and $E_{\mathrm{T}}$ is the energy of the triplet state (in $\mathrm{kJ} \cdot \mathrm{mol}^{-1}$ ); $\Phi_{\Delta}$, is the yield of singlet molecular oxygen production estimated based on $\Phi_{\Delta}=\frac{E_{h v}\left(1-\alpha^{\text {air }}\right)}{E_{\Delta}}-\frac{E_{\mathrm{S}}}{E_{\Delta}} \Phi_{\mathrm{F}}$ where $E_{\mathrm{S}}$ is the energy of singlet state $\left(\mathrm{kJ} \cdot \mathrm{mol}^{-1}\right)$ and $E_{\Delta}$ is the energy of the oxygen singlet state $\left(E_{\Delta}=94 \mathrm{~kJ} \cdot \mathrm{mol}^{-1}\right) ; S_{\Delta}$, is the fraction of dye's triplet state quenched by molecular oxygen

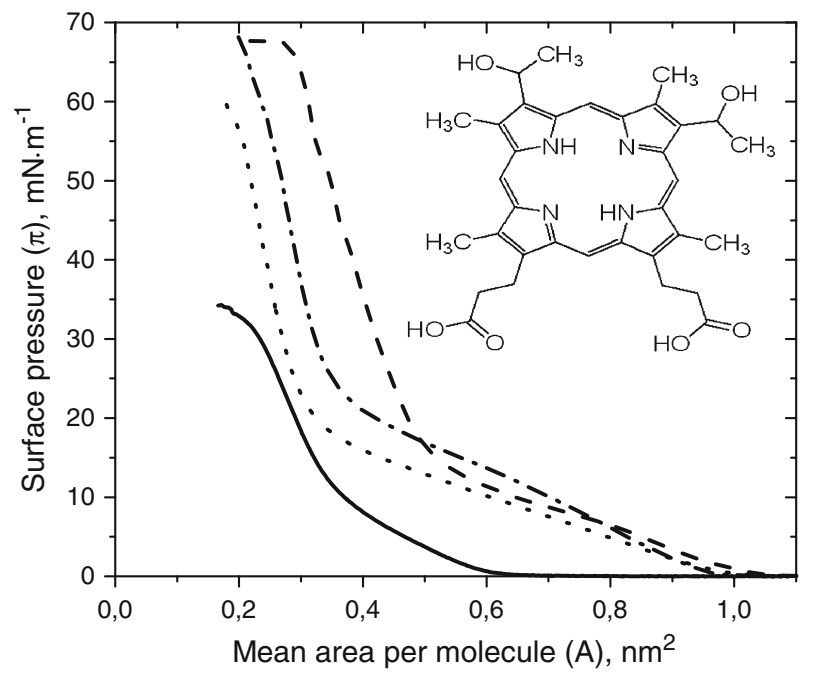

Fig. 1 Surface pressure-mean molecular area $(\pi-A)$ isotherms of HP and its mixture with DPPC (mole fraction (v:v) of components: 1:0 (solid), 0:1 (dashed), 1:1 (dotted), 1:4 (dashed-dotted)); inset-molecular structure of the dye

The compression $(\pi-A)$ isotherms of Langmuir monolayers of a dye-phospholipid, for the molar ratios studied, are given in Fig. 1. To characterize the interactions between the components and their miscibility, the compressibility modulus was calculated according to Eq. 3. The compressibility modulus versus the surface pressure is shown in Fig. 2. The presence of DPPC in the mixture has a stabilizing effect on the monolayer as indicated by the increasing slope of the lines (smaller angle between the 


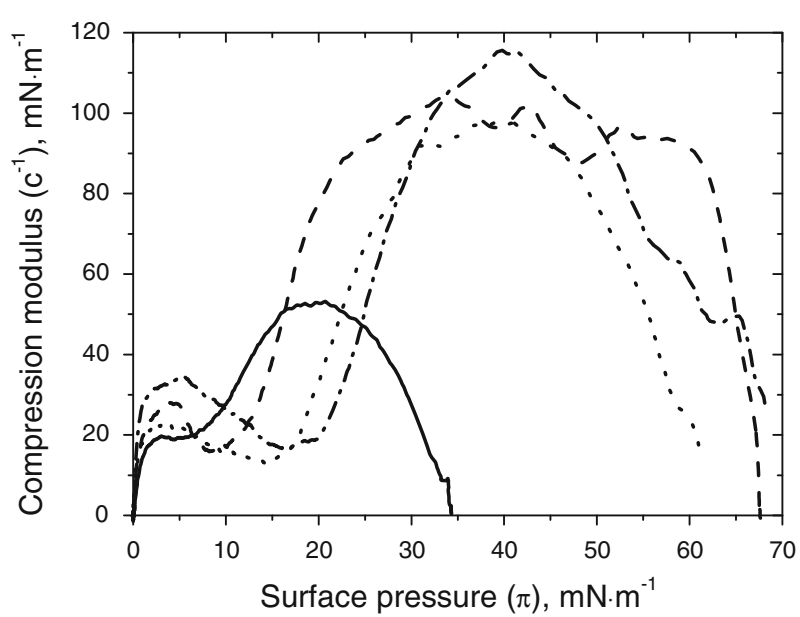

Fig. 2 Compression modulus versus surface pressure of the HP and DPPC mixtures studied (description of curves as in Fig. 1)

normal and the linear growing section of the isotherm) observed for the HP-DPPC mixtures relative to that for the isotherm recorded for pure HP (Fig. 1). The isotherm recorded for pure DPPC (Fig. 1) reveals a characteristic plateau, within which an liquid expanded-liquid condensed (LE-LC) phase transition takes place, described as the region of domain formation. In this region the hydrocarbon chains change the orientation from almost random- to highly-oriented, with increasing number of molecules in all-trans conformation [18]. For dye-lipid mixtures at the ratios of 1:4 and $1: 1$, the region of plateau is extended with respect to that for pure DPPC, which suggests that the LE-LC phase transition (Figs. 1, 2) takes place over a longer time to $\pi=17.5 \mathrm{mN} \cdot \mathrm{m}^{-1}$ for the area $0.40 \mathrm{~nm}^{2}$ and to $\pi=15 \mathrm{mN} \cdot \mathrm{m}^{-1}$ for the area $0.37 \mathrm{~nm}^{2}$.

An addition of DPPC causes an increase in the compressibility modulus so the layer becomes stiffer in the entire range of pressures studied. For the 1:4 and 1:1 mixtures, the maximum compressibility modulus increases over twice. For the 1:4 mixture, the compressibility modulus reaches a maximum of $\sim 115 \mathrm{mN} \cdot \mathrm{m}^{-1}$ at $\pi=$ $40 \mathrm{mN} \cdot \mathrm{m}^{-1}$, while for the $1: 1$ mixture, its maximum value is close to $95 \mathrm{mN} \cdot \mathrm{m}^{-1}$ at $\pi=39 \mathrm{mN} \cdot \mathrm{m}^{-1}$, i.e., lower than the maximum value for pure DPPC. The $\pi$ range of the maximum value of the compressibility modulus for DPPC is very wide, from $28 \mathrm{mN} \cdot \mathrm{m}^{-1}$ to $52 \mathrm{mN} \cdot \mathrm{m}^{-1}$, which is not observed for other molar fractions. One of the parameters permitting evaluation of the miscibility of the monolayer components is the position of the collapse point as a function of the molar fraction of the component added. By equating the second derivative of the isotherm to zero, the above dependence was determined (not shown). It was found to be linear with a high correlation coefficient $(0.944)$, which points to good miscibility of the compounds studied in monolayers.

An important thermodynamic parameter bringing information on the character of interactions between the component of a monolayer is the excess area determined 


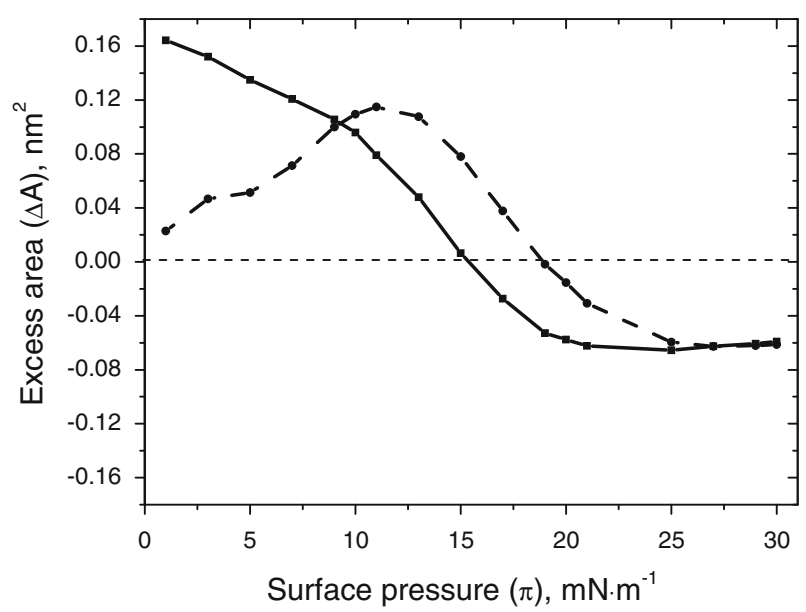

Fig. 3 Excess area versus surface pressure for HP and DPPC mixtures of 1:1 (solid) and 1:4 (dashed) mole fractions (v:v)

according to Eq. 1. The excess area (Fig. 3), for 1:1 and 1:4 mixtures of HP and $\mathrm{DPPC}$, takes positive values (evidencing repulsive interactions) for surface pressures below $15.0 \mathrm{mN} \cdot \mathrm{m}^{-1}$ and $17.5 \mathrm{mN} \cdot \mathrm{m}^{-1}$, respectively, and negative values (evidencing attractive interactions) for surface pressures from the values of $15.0 \mathrm{mN} \cdot \mathrm{m}^{-1}$ and $17.5 \mathrm{mN} \cdot \mathrm{m}^{-1}$ to about $30.0 \mathrm{mN} \cdot \mathrm{m}^{-1}$. For the systems studied, the excess area is saturated (at $-0.06 \mathrm{~nm}^{2}$, Fig. 3), which corresponds to stabilization of interactions in them.

Another important parameter is the excess Gibbs energy informing about the mutual interaction between the monolayer components and the influence of mixing on their character. Positive values of the excess Gibbs energy mean that the process of mixing is not thermodynamically preferred, so the mutual interactions between the monolayer components are weaker than those between the molecules in a single component monolayer. The excess Gibbs energy versus the molar fraction of DPPC in the mixtures studied is shown in Fig. 4. For the 1:1 mixture, it is lower than zero in the entire pressure range considered and suggests formation of an ideal mixture, while for the 1:4 system, it takes positive values only for surface pressures below $15.0 \mathrm{mN} \cdot \mathrm{m}^{-1}$ which can be related to the plateau on the compression isotherm corresponding to a much greater contribution of DPPC.

\section{Conclusions}

The interaction of HP was studied using different organic solvents which can modify the solvent-dye molecular interactions, and the Langmuir monolayers at air-water interface which can mimic biological membranes. The type of organic solvent used does not change the photophysical parameters describing the phototoxicity of the dye, and the results confirms high efficiency of triplet-triplet energy transfer between HP 


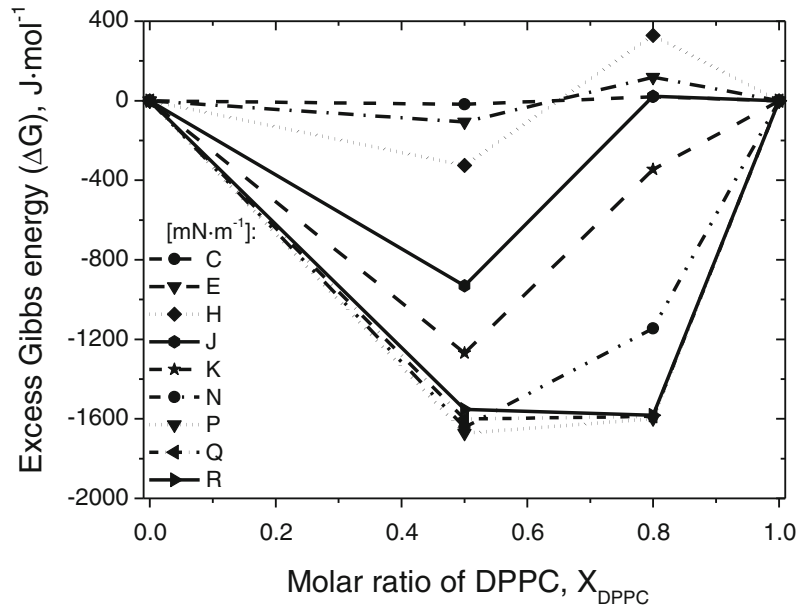

Fig. 4 Excess Gibbs energy as a function of DPPC mole fraction for different surface pressures

and oxygen, independent of the micro-environment, but the presence and phase state of lipids affect its thermodynamic parameters.

Analysis of the excess area and excess Gibbs energy has permitted identification of the possible regions of interaction stabilization in two-component monolayers and provided information on the dependence of miscibility of the monolayer components on its phase state and composition. It has been shown that HP makes stable monolayers on the subphase surface, and for the same contribution of molecules of both monolayer components, the process of HP-DPPC mixing is thermodynamically preferred leading to the formation of an ideal mixture.

Acknowledgments This study was supported by Poznan University of Technology (DS/2012 (JŁ, AD) and DS/MK/2012 (MK)). The authors thank Dr. eng. B. Jurzyk for her help in the LIOAS experiment performance.

Open Access This article is distributed under the terms of the Creative Commons Attribution License which permits any use, distribution, and reproduction in any medium, provided the original author(s) and the source are credited.

\section{References}

1. L. Caseli, F.S. Vinhado, Y. Iamamoto, M.E.D. Zaniquelli, Colloids Surf. A 229, 169 (2003)

2. M.T. Martin, I. Prieto, L. Camacho, D. Mobius, Langmuir 12, 26 (1996)

3. M.C. DeRosa, R.J. Crutchley, Coord. Chem. Rev. 233-234, 351 (2002)

4. M.L. Lomonosov, Khim.-Farm. Zh. 11, 2 (1977)

5. G.J. Smith, Photochem. Photobiol. 41, 2 (1985)

6. C. Tanielian, C. Schweitzer, R. Mechin, C. Wolff, Free Radical Biol. Med. 30, 2 (2001)

7. L.M. Moreira, F.V. dos Santos, J.P. Lyon, M. Maftoum-Costa, C. Pacheco-Soares, N. Soaresda Silva, Aust. J. Chem. 61, 10 (2008)

8. S. Bonneau, P. Morliere, D. Brault, Biochem. Pharmacol. 68, 7 (2004)

9. M. Korínek, D. Dědic, A. Molnár, A. Svoboda, J. Hála, J. Mol. Struct. 744-747, 727 (2005)

10. A.P. Ramos, C. Pavani, Y. Iamamoto, M.E. Zaniquelli, J. Colloid Interface Sci. 350, 1 (2010) 
11. M.C. Desroches, A. Kasselouri, M. Meyniel, P. Fontaine, M. Goldmann, P. Prognon, P. Maillard, V. Rosilio, Langmuir 20, 26 (2004)

12. B. Olejarz, B. Bursa, I. Szyperska, R.-M. Ion, A. Dudkowiak, Int. J. Thermophys. 31, 163 (2010)

13. M.I. Sandez Macho, A. Gil Gonzalez, A. Suarez Varela, J. Colloid Interface Sci. 235, 241 (2001)

14. E. Reddi, G. Jori, Rev. Chem. Intermed. 10, 241 (1988)

15. D. Bryce-Smith, Photochemistry, vol. 2, Specialist Periodical Report (Royal Society of Chemistry, London, 1971)

16. R.W. Redmond, J.N. Gamlin, Photochem. Photobiol. 70, 391 (1999)

17. S. Mathai, T.A. Smith, K.P. Ghiggino, Photochem. Photobiol. Sci. 6, 995 (2007)

18. G. Ma, H.C. Allen, Langmuir 22, 5341 (2006) 